\title{
Erratum to: Outcome of cochlear implantation in children with cochlear malformation
}

Dimitrios Rachovitsas • George Psillas •

Stefanos Triaridis $\cdot$ Victor Vital $\cdot$

Jiannis Constantinidis

Published online: 20 May 2014

(C) Springer-Verlag Berlin Heidelberg 2014

Erratum to: Eur Arch Otorhinolaryngol

DOI 10.1007/s00405-014-3059-6

Unfortunately, the article title was incorrect in the original publication. The corrected title should read:

Outcome of cochlear implantation in children with cochlear malformation

The online version of the original article can be found under doi:10.1007/s00405-014-3059-6.

D. Rachovitsas · G. Psillas $(\varangle) \cdot$ S. Triaridis $\cdot$ V. Vital First Academic ENT Department, Aristotle University of Thessaloniki, AHEPA Hospital, 1, Stilponos Kyriakidi St, 54636 Thessaloniki, Greece

e-mail: psill@otenet.gr

J. Constantinidis

Second Academic ENT Department, Aristotle University of Thessaloniki, Papageorgiou Hospital, Nea Eukarpia,

56429 Thessaloniki, Greece 